\title{
Lapurdum
}

Euskal ikerketen aldizkaria | Revue d'études basques |

Revista de estudios vascos | Basque studies review

$3 \mid 1998$

Numéro III

\section{Michel Morvan. Les origines linguistiques du basque.}

290 pp., Presses Universitaires de Bordeaux, 1996.

\section{Jacques Allieres}

\section{(2) OpenEdition}

1 Journals

Édition électronique

URL : https://journals.openedition.org/lapurdum/1746

DOI : 10.4000/lapurdum. 1746

ISSN : 1965-0655

Éditeur

IKER

Édition imprimée

Date de publication : 1 octobre 1998

Pagination : 315-317

ISBN : 2-84127-152-8

ISSN : $1273-3830$

Référence électronique

Jacques Allieres, « Michel Morvan. Les origines linguistiques du basque. », Lapurdum [En ligne], 3 | 1998, mis en ligne le 01 septembre 2010, consulté le 25 février 2022. URL : http://journals.openedition.org/ lapurdum/1746 ; DOI : https://doi.org/10.4000/lapurdum.1746 


\section{Michel Morvan. Les origines linguistiques du basque. \\ 290 pp., Presses Universitaires de Bordeaux, 1996.}

Voici un bel ouvrage, nécessaire et courageux. Reprenant sa thèse, Les origines linguistiques du basque: l'ouralo-altaïque, élaborée sous la direction du Professeur J.-B. ORPUSTAN et soutenue à Bordeaux en 1992, l'auteur se propose en effet de réexaminer les hypothèses qui tentent de rattacher le basque, généralement considéré comme un idiome isolé, à des familles connus autres que celles auxquelles on se réfère habituellement, à savoir la famille (?) caucasique et la famille chamitique. Dans une prudente et utile première partie (pp. 20-98), il résume l'historique du problème, soumettant à une revue critique les théories fantaisistes - préscientifiques ou tenant de l'élucubration -, ensuite les théories sérieuses : hypothèse finno-ougrienne, "basco-ibérisme », hypothèse indo-européenne (!), hypothèse africaine, enfin théorie caucasienne. Puis, abordant la matière proprement dite, il confronte dans sa seconde partie (pp. 99-158) le basque au substrat euro-sibérien plongeant d'abord «dans les profondeurs du passé » pour aborder ensuite plus précisément les langues paléoasiatiques (ghiliak, tchouktche, iénisséien), enfin dans sa troisième partie (pp. 159-266) à Youralo-altaïque, examiné avec une extrême minutie: 69 pages de morphologie, 31 de «phonétique et lexique».

Pour le romaniste et même l'indo-européanisant, le sémitisant, l'ouralisant ou l'altaïsant, l'entreprise peut paraître a priori très ambitieuse et vraisemblablement vouée à l'échec. Le premier dispose en effet de la langue-mère, le latin, dont il détermine l'évolution vers les langues-filles, les langues romanes, selon tout un arsenal de règles précises ; les quatre autres chercheurs ne possèdent peut-être pas les termini a quo de l'évolution comme le précédent, mais les attestations plus ou moins anciennes des langues de la famille permettent de reconstituer à la fois les transformations intervenues pour chacune d'entre elles et la forme hypothétique de la langue première dont elles sont issues suivant ces transformations. Face à ces types de recherche comparative, conformes au schéma traditionnel mais propres à des situations privilégiées, car les peuples parlant ces langues possédaient généralement l'écriture et nous ont laissé des témoignages anciens, échelonnés dans le temps et distribués dans l'espace, des linguistes s'attaquent aujourd'hui à des idiomes dépourvus de tradition écrite à la mesure de leur très longue histoire, s'efforçant néanmoins avec un certain succès de rétablir leur schéma évolutif et - bien entendu - le terminus a quo correspondant. Mais aucun stade intermédiaire ne peut jalonner les millénaires d'évolution possibles. Or le romaniste qui établit un rapport indiscutable entre les mots latins ILLU FRAXINU ou LEVO, LEVAS, LEVAT et leurs produits respectifs, gascon eth hrèische et roumain iau, iei, ia, ou encore l'indo-européanisant sûr de pouvoir démontrer que l'anglais wheel et le sanskrit cakram, ou encore le latin $d u o$ et l'arménien erku sont respectivement issus d'une même source, à savoir les étymons restitués $* \boldsymbol{k} \boldsymbol{w e k l o}$ - « roue », et $\boldsymbol{*} \boldsymbol{d w o}$ - « deux », opèrent en toute sécurité en dépit des apparences - celles-ci étant en revanche assez trompeuses pour laisser penser que l'anglais bad « mauvais » et le persan bad «id» sont frères, alors que leurs origines sont radicalement différentes. Et il en irait à peu près de même en domaine ouralien ou sémitique. Aussi des linguistes «traditionnels » de ce genre ne voient-ils pas sans un certain scepticisme mêlé d'inquiétude d'audacieux chercheurs tenter des comparaisons, des apparentements, des parallèles dans des domaines dont l'histoire nous est totalement inconnue, et se fonder sur des ressemblances actuelles, alors que chaque idiome concerné a vécu, postérieurement à la «communauté » primitive, une longue existence. Voilà pourquoi la tentative de Michel Morvan n'entraîne pas a priori l'adhésion des comparatistes « de la vieille école ». 
Néanmoins, l'auteur a conçu et réalisé dans son ouvrage un travail nécessaire, méritoire et important. Nécessaire parce que de telles hypothèses ont été émises dans un passé relativement lointain, à une époque où la science linguistique ne possédait ni les acquêts ni les « techniques » dont elle dispose aujourd'hui ; méritoire parce que le nombre des idiomes mis à contribution - 26 dans l'Index des «langues rares ou peu connues » (pp.281-284; voir aussi les deux cartes : p. 285 principales langues du Caucase, pp. 286 - 7 langues de l'Eurasie) - est considérable et implique un travail de recherche exemplaire; important parce qu'il nous permettra de sortir des impasses rencontrées jusqu'ici avec les théories traditionnelles, et de relier le problème basque avec les grandes perspectives « mondialistes » en honneur parmi les chercheurs, surtout - mais non exclusivement - américains.

Il ne saurait être question pour nous de reprendre avec un œil critique - dont nous prive notre ignorance des langues concernées - le long et minutieux travail de confrontation mené par notre auteur. Nous y avons trouvé un nombre considérable de rapprochements intéressants et souvent plausibles. Nos réticences à une adhésion complète résidant dans l'image évoquée ci-dessus d'une histoire phonétique complexe, exigeante et rigoureuse à la façon romane et indo-européenne, il nous suffit pour «y croire» d'admettre que les langues envisagées montrent, par contraste avec celle des deux domaines évoqués, une stabilité remarquable ; c'est ce que l'on a noté dans le domaine altaïque - les dialectes turcs, du Bosphore à la Sibérie, sont peu différenciés - et peut-être du basque, précisément, langue qui s'est très peu modifiée depuis les premiers textes. A partir de ces prémisses, on pourra accepter cette étonnante, fascinante gymnastique qui saute allègrement montagnes, mers et continents pour reconnaître des parentés. Cela pourrait être de l'ordre de «l'élucubration» dénoncée par Michel Morvan, si notre auteur ne se mouvait dans ce vaste univers avec une prudence et une précision dignes d'éloges. Evidemment, lorsque l'on songe que le structuralisme à ces débuts enseignait que la langue est un système clos « où tout se tient», on peut hésiter sérieusement à jeter de tels ponts qui, avec superbe font fi des systèmes et des structures cohérentes. Mais on retrouve effectivement dans cet ouvrage un souci des constructions partielles, des paradigmes homogènes qui fait accepter les audaces. Et la dialectologie comparée, qui confronte à grande échelle des langues de structure et d'origine diverses - comme c'est le cas dans l'ATLAS LINCUARUM EUROPAE (aire bien réduite par rapport à l'Eurasie !) -, nous enseigne bien que d'autres «structures », transversales celle-là, se manifestent avec autant d'évidence que les premières, dans un jeu de complémentarité tout à fait satisfaisant. Aussi applaudirons-nous à la conclusion, aussi prudente qu'audacieuse, par laquelle notre auteur clôt son beau travail. Non, le basque n'est plus isolé, et ses racines paraissent bien plonger dans les profondeurs de la préhistoire eurasiatique : reste aux chercheurs désireux de suivre Michel Morvan dans sa courageuse et passionnante quête à s'initier comme il l'a fait aux arcanes de l'euro-sibérien et de l'ouralo-altaïque. Ce ne leur sera pas aisé, car pour ce faire la matière est difficile, la bibliographie - polyglotte - considérable ; mais il est certain que l'enjeu mérite l'effort qu'avec un succès manifeste notre auteur a consenti, et dont la linguistique lui saura gré.

Jacques ALLIERES

Professeur émérite de l'Université de Toulouse-Le-Mirail UPRESA 5478 